\section{Emergenza-urgenza negli ospedali: siamo così diversi dall'America?}

\author{
Hospital-based emergency care: are we so different \\ from America?
}

\section{Egregio Direttore,}

in relazione ai due interessanti articoli a cura di Gardellini et al. e Nardi et al. [1,2] apparsi nel n. 1/2009 dell'Italian Journal of Medicine, che focalizzano l'attenzione sull'overcrowding nei reparti di Medicina, ci pare si possa estendere la riflessione anche ai Dipartimenti di emergenza.

I problemi del sistema emergenza-urgenza ospedaliero cosi come si sta sviluppando in Italia, a confronto con le più recenti analisi e riflessioni provenienti dall'esperienza statunitense, che risulta spesso tra i principali modelli di riferimento della pratica ospedaliera anche in Italia, non appaiono poi cosi differenti come talora si pensa.

Recentemente lo IOM (Institute of Medicine, organizzazione non profit americana che ha lo scopo istituzionale di produrre orientamenti indipendenti, obiettivi ed evidencebased per politici e amministratori della sanità pubblica e privata) ha sviluppato un'approfondita analisi del sistema dell'emergenza ospedaliera delineatosi nell'ultimo decennio negli Stati Uniti, e ha pubblicato un volume dall'emblematico titolo Hospital-Based Emergency Care: at the breaking point.

L'interessante e anche ironica premessa ricorda che, se si dovesse giudicare il sistema di emergenza sulla base della sua rappresentazione in noti telefilm, si avrebbe un'immagine di grande efficienza non corrispondente, però, alla situazione degli ospedali americani, la cui realtà è molto simile a quella che si può riscontrare in Italia, caratterizzata soprattutto dall'intenso affollamento del Pronto Soccorso.

L'analisi che viene effettuata del fenomeno sottolinea in particolare:

- il progressivo incremento degli accessi ai Dipartimenti di emergenza (specialmente nelle ore notturne e nei weekend, quando gli altri medici non sono disponibili), che hanno assunto anche funzioni vicarianti e suppletive rispetto a servizi territoriali meno efficienti, tutto ciò a fronte della riduzione complessiva delle risorse disponibili, anche in termini di capienza ospedaliera;

- la conseguente diffusione della pratica, nota come "boarding", di trattenere i pazienti nell'ambito dei
Dipartimenti di emergenza, spesso in letti improvvisati con riduzione della sicurezza, ritardi diagnostico-terapeutici, scarsa privacy ecc.

Non è possibile ignorare quanto la realtà descritta ricordi molto da vicino quella italiana.

Il documento, che è molto esteso e articolato e non ci proponiamo qui di analizzare nei dettagli, sottolineando in particolare che non esiste al momento una soluzione valida per tutti ed è quindi utile sperimentare soluzioni organizzative anche differenti, propone, per migliorare l'efficienza degli ospedali, le seguenti linee di indirizzo:

- modulazione delle risorse disponibili considerando le variabili legate ai picchi di massimo e minimo afflusso (queueing theory);

- sviluppo delle Unità di Osservazione Breve Intensiva 23 h; - coordinamento del flusso dei pazienti, a partire anche dalla considerazione che l'affollamento del Dipartimento di emergenza è essenzialmente un problema di tutto l'ospedale e non tanto o non solo del Dipartimento stesso.

Per quanto riguarda l'attuazione del terzo punto, lo strumento proposto è lo sviluppo, negli ospedali, di attività e funzioni di gestione proattiva delle risorse disponibili nell'intero ospedale (non soltanto nel Dipartimento di emergenza), spesso affidata alla figura dell' "hospitalist", cioè "un clinico la cui attività principale è l'assistenza medica generale dei pazienti ospedalizzati", secondo la definizione della Society of Hospital Medicine americana. Superando in tal modo il tradizionale paradigma che vede il Dipartimento di emergenza funzionare anche come "valvola di sfogo" per $i$ ritardi e le inefficienze ospedaliere, è possibile sviluppare invece una visione del Dipartimento altamente integrata $e$ interconnessa con l'intero Ospedale.

Viene quindi suggerito di monitorare, tramite la messa a punto di appositi standard, $i$ fenomeni di affollamento $e$ boarding nei Dipartimenti di emergenza.

In sintonia con le raccomandazioni dello IOM, presso il Johns Hopkins Bayview Medical Center di Baltimora (Maryland) è stata avviata un'esperienza orientata a organizzare nel modo migliore $i$ flussi dei pazienti all'interno dell'ospedale, fondata sulla figura dell'hospitalist in qualità di bed manager attivo [3].

I ricercatori hanno ottenuto una sensibile riduzione dei tempi di attesa e stazionamento nel Dipartimento di emergenza dei pazienti che successivamente sono stati ricoverati in ospedale (da 8 a 6 ore), la riduzione del $6 \%$ delle ore nelle quali il Dipartimento di emergenza veniva definito 
"sovraffollato" e del $27 \%$ di quelle in cui il Dipartimento di emergenza-accettazione non era in grado di ricoverare pazienti per mancanza di posti letto disponibili in ospedale. Poiché negli Stati Uniti, ancora di più che in Italia, quando un Dipartimento di emergenza è in difficoltà per sovraffollamento o mancanza di posti letto le ambulanze vengono dirottate presso altri ospedali, tali miglioramenti hanno comportato un incremento dell' $8,8 \%$ negli accessi presso il Dipartimento stesso.

Va altresi ricordato che, sebbene il dibattito su questi temi si stia sviluppando soprattutto negli Stati Uniti, la sua rilevanza è stata evidenziata anche da studi italiani, per certi versi pionieristici, come quello del gruppo di Carlo Alberto Perucci [4] che, indagando sul sovraffollamento degli ospedali romani durante le epidemie influenzali, concludeva: «Le degenze specialistiche reagiscono in modo insufficiente a un'emergenza modesta e prevedibile. Ogni crisi invernale di capienza ospedaliera è probabilmente il risultato più di una gestione deficitaria dei posti letto disponibili che di un eccessivo incremento della domanda».

Le considerazioni che ci sentiamo di poter formulare con riferimento anche alla politica sanitaria in Italia sono le seguenti:

- la pratica di aumentare le risorse ai Dipartimenti di emergenza per far fronte a situazioni di crisi può essere, di per sé, insufficiente e improduttiva;

- è necessario che il problema dell'emergenza-urgenza venga vissuto e "sentito" da tutto l'ospedale (e il territorio);

- è necessario attuare la massima integrazione tra i Dipartimenti di emergenza e le altre risorse ospedaliere, evitando soluzioni che tendano a consolidare dicotomie Ospedale/Pronto Soccorso.

Riteniamo pertanto opportuno che, come anche sostenuto dalla Gardellini e Nardi, vengano sviluppati strumenti di bed management, attività complessa e problematica che richiede soluzioni innovative, il cui interprete principale ci pare possa essere la figura di un medico internista con funzioni di hospitalist.

\section{Bibliografia}

[1] Gardellini A, Nardi R, Arienti V, Panuccio D, Bernardi R, Pedone V. Invecchiamento della popolazione, gestione dei pazienti, posti letto e sovraffollamento in ospedale. IJM 2009;3(1):35-43.

[2] Nardi R, Gardellini A, lori I. Sovraffollamento nei reparti di degenza e clinical risk management in Medicina Interna: soluzioni solamente strutturali o interventi sistemici sui processi assistenziali? IJM 2009;3(1):3-8.

[3] Howell E, Bessman E, Kravet S, Kolodner K, Marshall R, Wright S. Active bed management by hospitalists and emergency department throughput. Ann Intern Med 2008;149(11):804-11.

[4] Fusco D, Saitto C, Arcà M, Perucci CA. Influenza outbreaks and hospital bed occupancy in Rome (Italy): current management does not accommodate for seasonal variations in demand. Health Serv Manage Res 2006;19(1):36-43.

\footnotetext{
Enrico Haupt ${ }^{\mathrm{a}}$, Gaddo Flego $^{\mathrm{b}}$

${ }^{a}$ Medicina Interna, Dipartimento di Emergenza, ASL 4 Chiavarese Regione Liguria

${ }^{\mathrm{b}}$ Direzione Medica di Presidio Ospedaliero, ASL 4 Chiavarese Regione Liguria
}

$\mathrm{N}$ ella nostra esperienza, il concetto guida è stato proprio quello di considerare l'iperafflusso come un problema di tutto l'ospedale, coinvolgendo nel team di bed management il Dipartimento di emergenza, Medico, Chirurgico e Igienicoorganizzativo; con l'uso, poi, dello strumento informatico "Cruscotto di Pronto Soccorso", l'area dell'emergenza può inviare il paziente nel reparto di destinazione (all'interno del debito previsto e dei vari step della procedura di escalation), immediatamente, una volta terminato il percorso diagnostico-terapeutico. I tempi di attesa del trasferimento riconoscono, quindi, come unico limite la logistica dei trasporti interni. Esiste inoltre un atteggiamento proattivo che risulta in: a) una copertura, con un pool predefinito di risorse esterne, dell'eventuale mismatch fra afflusso atteso e attività delle Unità operative nei periodi di prevista crisi di risorse (epidemia influenzale, ondate di calore) e/o riduzione di attività; b) una gestione delle dimissioni protette unica metropolitana anch'essa in grado di adattarsi, entro certi limiti strutturali, a eventuali maggiori richieste.

Certamente questa organizzazione ha portato a un governo dei processi assistenziali e a una gestione più efficiente delle risorse di ricovero, com'è dimostrato dagli outcome delle varie unità medico-geriatriche. II bed management non rappresenta tuttavia, dal nostro punto di vista, la soluzione del problema, che è invece da ricercarsi nella gestione complessiva dell'intero percorso clinicoassistenziale del cittadino. Infatti, nonostante i progressi compiuti nell'organizzazione della presa in carico del cittadino da parte dei Distretti alla dimissione ospedaliera (anche se con evidenti differenze nei tempi dell'operatività fra ospedale e territorio), rimangono ancora assolutamente non governate l'urgenza ambulatoriale gestibile al di fuori dei Pronto Soccorso e la prevenzione degli episodi di scompenso delle malattie croniche, in gran parte causa del problema dei "frequent users" di Pronto Soccorso e di ricovero ospedaliero. L'overcrowding è forse risolvibile soltanto nell'ambito di una forte e complessiva azione di riordino dei processi produttivi in Sanità, azione che ponga al centro il cittadino/utente e intorno Unità operative ospedaliere e territoriali che riducano il più possibile le barriere fra l'una e l'altra, lavorando in un continuum organizzativo e clinico-assistenziale.

\author{
Aldina Gardellini ${ }^{\mathrm{a}}$, Roberto Nardi ${ }^{\mathrm{b}, *}$ \\ ${ }^{a}$ UOC Geriatria, Ospedale Maggiore, Bologna \\ ${ }^{\mathrm{b}}$ UoC Medicina Interna, Ospedale Maggiore, Bologna \\ ${ }^{*} E$-mail: r.nardi@ausl.bo.it
}

doi:10.1016/j.itjm.2009.06.006 